\title{
Prolonged Antibiotic Treatment does not Prevent Intra-Abdominal Abscesses in Perforated Appendicitis
}

\author{
K. van Wijck · J. R. de Jong • L. W. E. van Heurn • \\ D. C. van der Zee
}

Published online: 31 August 2010

(c) The Author(s) 2010. This article is published with open access at Springerlink.com

\begin{abstract}
Background Children with perforated appendicitis have a relatively high risk of intra-abdominal abscesses. There is no evidence that prolonged antibiotic treatment after surgery reduces intra-abdominal abscess formation. We compared two patient groups with perforated appendicitis with different postoperative antibiotic treatment protocols. Methods We retrospectively reviewed patients younger than age 18 years who underwent appendectomy for perforated appendicitis at two academic hospitals between January 1992 and December 2006. Perforation was diagnosed during surgery and confirmed during histopathological evaluation. Patients in hospital A received 5 days of antibiotics postoperatively, unless decided otherwise on clinical grounds. Patients in hospital B received antibiotics for 5 days, continued until serum C-reactive protein (CRP) was $<20 \mathrm{mg} / \mathrm{l}$. Univariate logistic regression analysis was performed on intention-to-treat basis. $p<0.05$ was considered significant.
\end{abstract}

K. van Wijck $(\bowtie) \cdot$ L. W. E. van Heurn

Department of Surgery, Maastricht University Medical Center, P.O. Box 616, 6200 Maastricht, MD, The Netherlands

e-mail: k.vanwijck@ah.unimaas.nl

J. R. de Jong

Department of Pediatric Surgery, University Medical Center, Amsterdam, The Netherlands

D. C. van der Zee

Department of Pediatric Surgery, Wilhelmina Children

University Medical Center, Utrecht, The Netherlands

L. W. E. van Heurn

Department of Pediatric Surgery, Maastricht University Medical Center, Maastricht, The Netherlands
Results A total of 149 children underwent appendectomy for perforated appendicitis: 68 in hospital A, and 81 in hospital B. As expected, the median (range) use of antibiotics was significantly different: 5 (range, 1-16) and 7 (range, 2-32) days, respectively $(p<0.0001)$. However, the incidence of postoperative intra-abdominal abscesses was similar $(p=0.95)$. Regression analysis demonstrated that sex (female) was a risk factor for abscess formation, whereas surgical technique and young age were not.

Conclusions Prolonged use of antibiotics after surgery for perforated appendicitis in children based on serum CRP does not reduce postoperative abscess formation.

\section{Introduction}

Appendicitis is the most common acute condition that requires surgery in children. Yet there is no consensus regarding the optimal antimicrobial treatment for children with uncomplicated or complicated appendicitis [1-3]. Appendicitis has a broad spectrum of severity and therapy should be categorized accordingly. Of all children with appendicitis, those with perforated appendicitis are especially prone to develop intra-abdominal abscesses. Children with perforated appendicitis are usually treated with intravenous antibiotics. However, there is no common opinion about the time period during which antibiotic treatment should be given. Nor is it clear whether prolonged antibiotic treatment reduces the incidence of intraabdominal abscess formation. Recently, the Therapeutic Agents Committee of the Surgical Infection Society published guidelines on antimicrobial therapy for children with appendicitis in general, including recommendations for treatment of children operated on for perforated appendicitis, based on the available literature [3]. However, little or 
no evidence exists to decide whether prolonged use of postoperative antibiotic treatment prevents intra-abdominal abscess formation in this particular patient group. Therefore, the goal of this study was to investigate whether prolonged use of antibiotics after surgery reduces intraabdominal abscess formation in children with perforated appendicitis.

\section{Patients and methods}

Patients and definition of perforated appendicitis

Patients younger than age 18 years operated on for perforated appendicitis at two academic pediatric surgical departments (A and B) between January 1st, 1992 and December 31st, 2006 were included, and patient data were reviewed retrospectively.

One patient was excluded from the analysis because of a urinary tract infection, which required prolonged antibiotic treatment. Perforation was defined as a non-iatrogenic lesion in the appendix. It was diagnosed during surgical intervention and confirmed postoperatively through histopathological evaluation. All resected specimens were histopathologically evaluated.

\section{Antimicrobial treatment regimens}

In all children, antibiotic treatment was started with a single intravenous dose before the incision was made. The antibiotic treatment protocol for perforated appendicitis with intravenously administered Augmentin (amoxicillin clavulanate) was similar in both hospitals for the first 5 days after surgery. In hospital B, this antibiotic therapy was complemented with gentamicin. After 5 days, antibiotic treatment was discontinued in hospital A (group A), unless staff decided otherwise on clinical grounds (for example temperature, patient's oral intake). In hospital B (group B), serum C-reactive protein (CRP) levels were determined after 5 days of antibiotic treatment on a daily basis at the clinical chemistry laboratory, and only Augmentin was continued until CRP levels decreased to $<20 \mathrm{mg} / \mathrm{l}$. In a minority of cases, a positive culture gave impetus to switch or complement the standard antibiotic treatment.

\section{Definition of intra-abdominal abscess}

Intra-abdominal abscess was strictly defined as a symptomatic collection of fluid within the abdominal cavity that was diagnosed on ultrasound, computed tomography, or at surgical intervention, and which was secondary to initial appendectomy.
Statistical analysis

All statistical analyses were performed on an intention-totreat basis using GraphPad Prism version 4.00, 2003 (GraphPad Software, Inc.) and SPSS version 15.0, 2006 (Lead Technologies, Inc.). Numeric data of both treatment groups were mostly compared using the Student's $t$ test, and data were presented as mean (standard error of the mean, SEM). When data were not normally distributed, the Mann-Whitney $U$ test was used and data were expressed as median (range). Univariate logistic regression analyses were used to evaluate the influence of potential confounders, such as age, sex, hospital, and surgical technique. A $p$ value $<0.05$ was considered significant.

\section{Results}

Patients

During the period studied, 620 children underwent surgery for acute appendicitis in one of the two participating centers; 149 (24\%) of these children underwent appendectomy for perforated appendicitis: 68 in hospital A (group A), and 81 in hospital B (group B; Table 1). The median age was 11 (range, 1-17) years in group A versus 9 (range, 0-17) years in group B. This difference was significant $(p<0.001)$. The patient, treatment, and outcome characteristics of both groups are listed in Table 1.

\section{Follow-up}

Three children were lost to follow-up after successful discharge from the hospital. One of these patients was admitted to hospital A during a trip abroad and returned home after being discharged, one patient (hospital A) had postoperative outpatient appointments at another hospital and was lost to follow-up, and one patient (hospital B) never kept her appointment at the outpatient clinic and did not respond to calls. These children were not excluded from the analysis. Patient follow-up was complete for 146 children (98\%). No other data were missing.

\section{Surgical procedure}

Seventy-two (48\%) children underwent laparoscopic appendectomy. Open appendectomy was performed in 77 $(52 \%)$ children (Table 1$)$. Because both participating centers are university hospitals, surgical procedures were performed by multiple surgeons and residents under supervision. Surgical protocols revealed no specific differences. 
Table 1 Characteristics of group A and group B

Data are numbers with percentages in parentheses unless otherwise indicated

Table 2 Analysis of potential risk factors

$O R$ odds ratio; $C I$ confidence interval

\begin{tabular}{llcc}
\hline Demographic & Group A $(n=68)$ & Group B $(n=81)$ & $p$ value \\
\hline Male & $43(63)$ & $39(48)$ & 0.11 \\
Age (year), median (range) & $11(1-17)$ & $9(0-17)$ & $<0.001$ \\
Surgical technique & & & \\
$\quad$ Laparoscopic appendectomy & $21(31)$ & $51(63)$ & $<0.01$ \\
$\quad$ Open appendectomy & $47(69)$ & $30(37)$ & $<0.01$ \\
Use of antibiotics, median (range) & $5(1-16)$ & $7(2-32)$ & $<0.0001$ \\
Abscess formation & $13(19)$ & $16(20)$ & 0.95 \\
\hline
\end{tabular}

\begin{tabular}{llll}
\hline Variable & \multicolumn{3}{l}{ Univariate logistic regression analysis } \\
\cline { 2 - 4 } & $p$ value & OR & $95 \%$ CI \\
\hline Sex (male) & 0.042 & 0.422 & $0.183-0.971$ \\
Age (year) & 0.306 & 1.057 & $0.951-1.175$ \\
Hospital (hospital A) & 0.922 & 0.96 & $0.425-2.17$ \\
Surgical technique (open technique) & 0.412 & 0.711 & $0.315-1.606$ \\
\hline
\end{tabular}

\section{Antibiotic treatment}

Median duration of antibiotic treatment was significantly shorter in group A than in group B: 5 (range, 1-16) days versus 7 (range, 2-32) days ( $p<0.0001$; Table 1), which was expected due to the difference in treatment protocols. In group A, 10 patients received antibiotic treatment for more than 5 days. Only 4 of these patients had an intraabdominal abscess. In group B, 54 patients received antibiotic treatment for more than 5 days.

In group B, all (100\%) children received gentamicin as part of the standard antibiotic regimen for perforated appendicitis, whereas in group A only 13 (18.8\%) children received gentamicin in addition to standard Augmentin. In 3 patients of group A, a positive culture of abdominal fluid gave impetus to add metronidazole to the standard antibiotic treatment. Patients were discharged when antibiotic treatment was complete, serum white blood count had normalized, and patients were afebrile and able to tolerate liquid foods at least.

Serum white blood counts at completion of antibiotic treatment were not available from all patients included, but the mean white blood count determined in $63.2 \%$ of patients was $11.7(0.76) \times 10^{9}$ white blood cells per liter of blood.

\section{Intra-abdominal abscess formation}

Twenty-nine (19.5\%) of all children operated on for perforated appendicitis developed an intra-abdominal abscess: $13(18.8 \%)$ in group A, and $16(19.8 \%)$ in group B $(p=0.95$; Table 1$)$. The mean age of the children who developed an intra-abdominal abscess postoperatively was
9.8 (0.7) years, compared with $9.5(0.3)$ years in the total group of patients. The age of the children with an abscess was not significantly different between the two hospitals (10 (range, 3-14) years in hospital B compared with 11 (range, 6-17) years in hospital A, $p=0.142$ ). Univariate logistic regression analysis demonstrated that intraabdominal abscesses occurred more often in girls ( $p=0.042$; odds ratio (OR), $0.422 ; 95 \%$ confidence interval (CI), 0.183-0.971). No other independent risk factors (such as age, hospital, and surgical technique) were identified for the development of an intra-abdominal abscess (Table 2).

\section{Treatment of intra-abdominal abscesses}

Whereas in two $(6.9 \%)$ patients, the intra-abdominal abscess resolved without intervention, the standard antibiotic treatment was prolonged in three $(10.3 \%)$ other children, without performing a surgical procedure to eliminate the abscess. Fifteen $(51.7 \%)$ children were treated by incision and drainage of the abscess - transanally or percutaneously. Nine $(31.0 \%)$ children underwent formal surgery for abscess drainage and lavage a total of 14 times (mean per child, 1.4).

\section{Discussion}

There is no single evidence-based antibiotic treatment strategy for uncomplicated or perforated appendicitis, particularly because there is no indisputable evidence for any optimal antimicrobial therapy after surgery for appendicitis in children [3]. Despite a marked decline in 
associated mortality during the past 50 years, perforation and complication rates remain unchanged, because they are influenced strongly by factors untouched by the intervening therapeutic advances. Scanty data exist about optimal duration of postoperative antibiotic treatment, whereas it is important to treat patients with adequate antimicrobial therapy and to avoid overtreatment.

Some authors advocate a predefined duration of antimicrobial treatment, whereas others discontinue antibiotics depending on the patient's clinical signs regardless of length of therapy. Unfortunately, studies that have investigated antibiotic regimens and included duration of postoperative antibiotic treatment as a point of interest often included children with perforated as well as gangrenous appendicitis [4-9], thereby assembling a different population, with a lower rate of postoperative intra-abdominal abscesses. The recently published guidelines from the Therapeutic Agents Committee of the Surgical Infection Society for antimicrobial therapy in children recommend that, with respect to perforated appendicitis, treatment duration should be based on the patient's response to treatment and intravenous therapy should be continued until the patient is afebrile, has normal white blood cell counts-in brief, has no symptoms of infection left at all [3]. However, these recommendations are partly based on studies that included children with gangrenous appendicitis. Gangrenous appendicitis is a different entity with an intact intestinal lining that should be treated by resection, with only $24 \mathrm{~h}$ of postoperative antibiotics $[10,11]$. In the current study, only children with perforated appendicitis were included to narrow the spectrum of the disease and to examine the influence of antibiotic therapy in a patient population that is most prone to develop intra-abdominal abscesses. By selecting this predisposed group, we designed to enlarge the possible effects of the different antibiotic treatment protocols.

Duration of antibiotic treatment could be determined by relying on criteria, such as normalized acute phase proteins (e.g., CRP), white blood count, and normal findings at bedside examination. However, in theory, this could lead to excessive use of antibiotics, because abnormal findings may be caused by an inflammatory response rather than an infection [10]. In the current study, the serum white blood count was not a reliable determinant to decide whether to discharge a patient. Snelling et al. presented a clear overview of abundant (often unspecified) criteria upon which antibiotic treatment is modified or discontinued in the current practice [4]. Opponents of the use of such criteria plead that management of infections should include patient support and antimicrobial therapy, but above all it should include an intervention to deal with the underlying process (e.g., drainage of an intra-abdominal abscess) [10-12]. Only when infections are not easily controllable, prolonged antibiotic therapy may be required. Thus, persistence of inflammation beyond the time of antibiotic therapy is not an indication to continue, restart, or change the antibiotic, but it is a hint that a treatable source of infection might be present, in absence of which signs of inflammation will remit spontaneously.

The current study was set up as a retrospective study with the primary purpose to compare two antibiotic treatment regimens in two patient groups with an equally high risk of abscess formation postoperatively. However, whereas the multicenter design of the study enabled us to weigh both regimens, it also increased the amount of variability between both treatment groups. For example, the mean age in hospital B is significantly lower than in hospital A. It is reported in literature that appendicitis may manifest itself more severe in younger children [13, 14]. Therefore, in theory, it is possible that the negative impact of age blurs the beneficial effect of prolonged antibiotic treatment, and the same assumption can be made for the sex difference and surgical approach, even though comparison of the two surgical protocols revealed no differences in general. To exclude the influence of potentially confounding factors, such as age, sex, and surgical technique on abscess formation, each factor has been taken into account in multivariate regression risk analysis, which revealed that no other potential risk factors apart from female sex predispose to intra-abdominal abscess development postoperatively. Because both participating centers are university hospitals, surgical procedures were performed by multiple surgeons and residents under supervision. This explains the relatively high risk of open surgery, because to enable residents to learn the standard open procedure, after diagnosis of a positive appendix using a scope without inflating the abdomen, open appendectomy may have been performed when the resident was not yet trained to perform laparoscopic appendectomy. It would have been more elegant to compare treatment groups within one hospital to eliminate the risk of confounding variables.

Both patient groups were identical regarding the onset and type of antibiotics. Although children in hospital B received gentamicin as part of the standard antibiotic protocol, and only $18.8 \%$ of children in hospital A did, we believe that we compared two adequate and equivalent antibiotic regimens. As stated, our purpose was to compare the duration of adequate antibiotic treatment to determine whether a more aggressive antibiotic approach would reduce the risk of intra-abdominal abscess formation. Whereas the postoperative intra-abdominal abscess rate found in the current study is relatively high, it is still within the range reported in previous studies for the pediatric population operated on for perforated appendicitis (3-24\%) [6, 15-18]. Using a strict definition for perforated appendicitis elevates the abscess rate within this population [17], and because 
clear definitions for both perforated appendicitis and intraabdominal abscess were applied in the current study, this could explain the high abscess rates that were found.

Not minifying the limitations of the current study, the clear results imply that prolonged antibiotic treatment after surgery for perforated appendicitis based on serum CRP level does not reduce the incidence of intra-abdominal abscesses. These findings are in agreement with the study by Henry et al. who also found no correlation between length of postoperative antibiotic treatment and abscess formation in children operated on for perforated appendicitis [15], and with the study by Lelli et al. who reviewed a historical cohort of children [19].

We would like to suggest a randomized, controlled trial to determine the optimal duration of postoperative antibiotic treatment for children with perforated appendicitis.

\section{Conflict of interest None.}

Open Access This article is distributed under the terms of the Creative Commons Attribution Noncommercial License which permits any noncommercial use, distribution, and reproduction in any medium, provided the original author(s) and source are credited.

\section{References}

1. Chen C, Botelho C, Cooper A et al (2003) Current practice patterns in the treatment of perforated appendicitis in children. J Am Coll Surg 196:212-221

2. Newman K, Ponsky T, Kittle K et al (2003) Appendicitis 2000: variability in practice, outcomes, and resource utilization at thirty pediatric hospitals. J Pediatr Surg 38:372-379

3. Nadler EP, Gaines BA (2008) The Surgical Infection Society guidelines on antimicrobial therapy for children with appendicitis. Surg Infect (Larchmont) 9:75-83

4. Snelling CM, Poenaru D, Drover JW et al (2004) Minimum postoperative antibiotic duration in advanced appendicitis in children: a review. Pediatr Surg Int 20:838-845

5. Taylor E, Dev V, Shah D et al (2000) Complicated appendicitis: is there a minimum intravenous antibiotic requirement? A prospective randomized trial. Am Surg 66:887-890
6. Hoelzer DJ, Zabel DD, Zern JT et al (1999) Determining duration of antibiotic use in children with complicated appendicitis. Pediatr Infect Dis J 18:979-982

7. Keller MS, McBride WJ, Vane DW et al (1996) Management of complicated appendicitis. A rational approach based on clinical course. Arch Surg 131:261-264

8. Neilson IR, Laberge JM, Nguyen LT et al (1990) Appendicitis in children: current therapeutic recommendations. J Pediatr Surg 25:1113-1116

9. St Peter SD, Tsao K, Spilde TL et al (2008) Single daily dosing ceftriaxone and metronidazole versus standard triple antibiotic regimen for perforated appendicitis in children: a prospective randomized trial. J Pediatr Surg 43:981-985

10. Schein M, Wittman DH, Lorenz W (1996) Duration of antibiotic treatment in surgical infections of the abdomen. Forum statement: a plea for selective and controlled postoperative antibiotic administration. Eur J Surg Suppl 576:66-69

11. Schein M, Assalia A, Bachus H (1994) Minimal antibiotic therapy after emergency abdominal surgery: a prospective study. Br J Surg 81:989-991

12. Nathens AB, Rotstein OD (1996) Antimicrobial therapy for intraabdominal infection. Am J Surg 172:1S-6S

13. Bratton SL, Haberkern CM, Waldhausen JH (2000) Acute appendicitis risks of complications: age and Medicaid insurance. Pediatrics 106:75-78

14. Mallick MS (2008) Appendicitis in pre-school children: a continuing clinical challenge. A retrospective study. Int J Surg 6:371-373

15. Henry MC, Walker A, Silverman BL et al (2007) Risk factors for the development of abdominal abscess following operation for perforated appendicitis in children: a multicenter case-control study. Arch Surg 142:236-241

16. Almond SLM, Roberts M, Joesbury V et al (2008) It is not what you do, it is the way that you do it: impact of a care pathway for appendicitis. J Pediatr Surg 43:315-319

17. St Peter SD, Sharp SW, Holcomb DW 3rd et al (2008) An evidence-based definition for perforated appendicitis derived from a prospective randomized trial. J Pediatr Surg 43:2242-2245

18. Henry MC, Gollin G, Islam S et al (2000) Matched analysis of nonoperative management vs. immediate appendectomy for perforated appendicitis. J Pediatr Surg 42:19-23 discussion 23-24

19. Lelli JL Jr, Drongowski RA, Raviz S et al (2000) Historical changes in the postoperative treatment of appendicitis in children: impact on medical outcome. J Pediatr Surg 35:239-244 discussion 244-245 